\title{
An in-vitro model of colonisation resistance to Clostridium difficile infection
}

\author{
S. P. BORRIELLO and FIONA E. BARCLAY
}

Division of Communicable Diseases, Clinical Research Centre, Harrow, Middlesex, England HA1 3UJ

\begin{abstract}
Summary. To investigate the importance of the normal gut flora in preventing the establishment of Clostridium difficile in vivo we have developed an in-vitro test system based on growth in faecal emulsions. Growth of $C$. difficile and cytotoxin production are inhibited in faecal emulsions from healthy adults, but not in sterilised emulsions; the importance of viable bacteria in the inhibitory system is evident. Generally, faecal emulsions derived from infants, children and geriatric patients were less inhibitory than those from healthy adults. Those from bottle-fed infants were significantly less inhibitory than those from breast-fed infants. Decreased levels of cytotoxin in the latter group were attributed to the acidic $p \mathrm{H}$ of the stools. With the different patient groups studied, faecal samples not inhibitory to $C$. difficile in vitro were obtained from $21 \%$ of patients with antibiotic-associated diarrhoea, $33 \%$ of those taking antibiotics but who did not have diarrhoea, $18.7 \%$ of those with diarrhoea unassociated with antibiotics, and $79 \%$ of those with $C$. difficile-mediated diarrhoea. In some cases inhibition was due to low faecal $p \mathrm{H}$, as in some infants, and in others to other filterable substances. The degree of inhibition could not be linked to specific volatile fatty acids or enzymes.
\end{abstract}

\section{Introduction}

Clostridium difficile is a well established cause of pseudomembranous colitis (PMC), as well as of many cases of antibiotic-associated diarrhoea (AAD) and some cases of diarrhoea unrelated to antibiotic therapy (Bartlett et al., 1978; George et al., 1978; Larson et al., 1978; Borriello and Larson, 1981; Brettle et al., 1982). There is much evidence to imply that a stable normal gut flora acts as a natural barrier to infection with $C$. difficile (Bowden et al., 1981; Wilson et al., 1981; Borriello and Barclay, 1984; Schwan et al., 1984), and that infection with $C$. difficile generally requires recent or current antimicrobial therapy to disrupt the gut flora and create an intestinal environment conducive to growth of $C$. difficile and production of its toxins. In the Syrian hamster model of PMC, disease is induced by the administration of antibiotics which disrupt the normal gut flora and subsequent exposure to pathogenic strains of $C$. difficile; neither antibiotic treatment nor exposure to $C$. difficile alone is sufficient to produce disease if animals are housed in protected (i.e., C. difficile-free) environments (Larson et al., 1980). Many attempts have been made to identify the individual components of the faecal flora that are antagonistic to $C$. difficile in vitro (Rolfe et al., 1981; Barclay and Borriello, 1982

Received 19 Jul. 1985; revised version accepted 28 Aug. 1985. and 1984; Malamou-Ladas and Tabaqchali, 1982). The significance of this type of study is obviously limited, as the conditions used relate poorly to those found in vivo. Recent work has shown that in most cases so far examined the antagonism noted between various components of the faecal flora and $C$. difficile is attributable to the acidic conditions produced; the inhibition is lost if the experiments are conducted in a medium buffered to neutrality or in sterilised faecal emulsions (Borriello and Barclay, unpublished observations).

In view of the interest in restoration of a normal faecal flora by use of faecal enemas as a means of treating troublesome cases of $C$. difficile gastrointestinal infections (Bowden et al., 1981; Schwan et al., 1984), we investigated the possibility of developing an in-vitro model of resistance to gastrointestinal infection with $C$. difficile based on the growth of, and toxin production by, $C$. difficile in emulsions prepared from the faeces of various patient groups and of healthy volunteers. This model was used to assess the relative roles of viable bacteria and cell-free components in inhibiting the growth of $C$. difficile. The model was used to compare different patients and to compare stools from healthy volunteers of different ages. Furthermore, an attempt was made to identify a chemical marker that might correlate with the relative inhibition of these faecal emulsions for growth of $C$. 
difficile and to assess the role of volatile fatty acids in this inhibition.

\section{Materials and methods}

\section{Sources of bacteria}

The strains of $C$. difficile were isolated in this laboratory from faeces. Strains B-1 and P-1 were isolated from patients with PMC. Strains T-1 and MA were isolated from asymptomatic infants, strain BL from an asymptomatic geriatric patient, and strain BT from the environment of a neonatal unit. All of these strains produced both toxin A (enterotoxin) and toxin B (cytotoxin). Strain M-1 was isolated from an adult with diarrhoea due to Shigella sonnei, and strain K-1 from an asymptomatic infant; both of these strains were non-toxigenic. After an initial experiment in which the eight strains of $C$. difficile were used, strain B-1 was selected for use throughout these investigations.

\section{Source of specimens}

Stool samples from healthy adults were obtained from colleagues at the Clinical Research Centre. Samples were also acquired from their children. Stools from geriatric patients who were not receiving antibiotic therapy were collected from the geriatric wards at Northwick Park Hospital (NPH). Stools from bottle-fed and breast-fed babies were obtained from the maternity unit at NPH. Samples from patients with AAD were forwarded to us from several hospitals throughout the United Kingdom to be screened for $C$. difficile and its cytotoxin as part of a national study of antibiotic-associated diarrhoea and colitis. Samples from patients with diarrhoea unassociated with antibiotic therapy were obtained from the Microbiology Department at NPH. Hamster caecal material was obtained from adult Syrian hamsters supplied from the National Institute for Medical Research. All faecal and caecal specimens were screened routinely for $C$. difficile and its cytotoxin before use (Borriello and Honour, 1981).

\section{Incubation conditions}

Anaerobic incubation was at $37^{\circ} \mathrm{C}$ in an atmosphere of $\mathrm{CO}_{2} 10 \%, \mathrm{H}_{2} 10 \%$ and $\mathrm{N}_{2} 80 \%$, in an anaerobic incubator housed in an anaerobic cabinet (Forma Scientific, Marietta, OH, USA).

\section{Preparation of inoculum}

Strains of $C$. difficile were inoculated from Columbia Blood Agar (Difco) into $10 \mathrm{ml}$ of Brain Heart Infusion Broth (Difco) containing L-cysteine $\mathrm{HCl} 0.05 \% \mathrm{w} / \mathrm{v}$ and sodium formaldehyde sulphoxylate $0.03 \% \mathrm{w} / \mathrm{v}$ as reducing agents (BHI broth), and incubated anaerobically for $18 \mathrm{~h}$. This culture was added to $100 \mathrm{ml}$ of BHI broth, incubated anaerobically for $6 \mathrm{~h}$, centrifuged at $4500 \mathrm{rpm}$ at $4^{\circ} \mathrm{C}$ for $15 \mathrm{~min}$, and the pellet washed twice by resuspending in $100-\mathrm{ml}$ volumes of fresh BHI broth. The pellet was finally suspended in $10 \mathrm{ml}$ of fresh BHI broth to provide the standard inoculum for the following experiments. The ratio of inoculum to faecal emulsion was always $1: 100 \mathrm{v}: \mathrm{v}$. Total viable counts and spore estimations (see below) were performed on these $C$. difficile suspensions which invariably contained $c .10^{8} \mathrm{cfu} / \mathrm{ml}$ and c. $10^{2}$ spores $/ \mathrm{ml}$.

\section{Preparation of faecal emulsions and treatment}

Stools of a normal consistency obtained from healthy subjects were all diluted 1 in $20 \mathrm{w} / \mathrm{v}$ in sterile distilled water which had been pre-reduced in an anaerobe cabinet before use. The faeces were mixed under anaerobic conditions on a vortex mixer to produce an even suspension. Specimens from patients with diarrhoea ranged from a fairly normal consistency to extremely liquid. These were diluted according to their fluidity to give a suspension roughly comparable to a 1 in $20 \mathrm{w} / \mathrm{v}$ dilution of normal stool. From a standardised suspension of $C$. difficile, $0 \cdot 1 \mathrm{ml}$ was inoculated into a $10-\mathrm{ml}$ volume of the faecal emulsion under test and mixed well before anaerobic incubation at $37^{\circ} \mathrm{C}$ for $72 \mathrm{~h}$. Samples were taken at 0 , 24,48 and $72 \mathrm{~h}$ for total viable count and spore count, cytotoxin estimation and $p \mathrm{H}$ measurements.

\section{Cytotoxin detection}

Cytotoxin in faecal or caecal emulsions was detected by filtering a sample of the emulsion through a $0.45-\mu \mathrm{m}$ membrane (Gelman Sciences Ltd, Northampton) and applying the filtrate to a monolayer of African green monkey kidney (VERO) cells. Cell-free filtrates derived from 48-h anaerobic cultures of $C$. difficile in Chopped Meat-Carbohydrate (CMC) Broth (Southern Group Laboratories, Hither Green, London) were similarly tested for the presence of cytotoxin. In both cases any cytopathic effect observed was neutralised (at the lowest dilution) with $C$. sordelli antitoxin (Wellcome Research Laboratories, Beckenham, Kent).

\section{Micromethod for quantitation of $C$. difficile in faecal emulsions}

In view of the large numbers of faecal emulsions that require quantitative analysis for $C$. difficile, we developed a micromethod that allowed the simultaneous dilution of up to eight faecal emulsions. In a microtitration tray (Sterilin Ltd, Middlesex), $200 \mu \mathrm{l}$ of each of eight faecal emulsions were added to the first vertical column, and $100 \mu \mathrm{l}$ of each into the seventh vertical column. All the remaining wells were filled with $180 \mu \mathrm{l}$ of BHI broth from a multi-channel pipette. Absolute ethanol $(100 \mu \mathrm{l})$ was added to the faecal emulsion in the seventh vertical column of the tray, mixed well, covered, and left for $1 \mathrm{~h}$ to select for spores (Koransky et al., 1978). Five serial tenfold dilutions of all the faecal emulsions were performed simultaneously with a multichannel pipette. Dilutions of 
the faecal emulsions were readily seeded on to agar medium by fitting pipette tips on to three alternate points of the multi-channel pipette and simultaneously seeding $10-\mu \mathrm{l}$ volumes of the $10^{5}, 10^{3}$ and $10^{1}$ dilutions on to one half of the agar medium and, retaining the same pipette tips, moving one dilution step down the series and seeding $10-\mu$ l samples of the $10^{4}$ and $10^{2}$ dilutions and the undiluted emulsion on to the other half of the plate. For total counts of $C$. difficile the cycloserine-cefoxitin selective medium described by Borriello and Honour (1981) was used, and for the alcohol-treated samples, Columbia blood agar was used. After anaerobic incubation for $48 \mathrm{~h}$, the seeded plates were screened for colonies of $C$. difficile by their characteristic fluorescence under long-wave ultraviolet light (George et al., 1979; Borriello and Honour, 1981); colony and cell morphology on nonselective medium (Borriello and Honour, 1981); and their characteristic volatile fatty-acid products of metabolism after growth in CMC broth (Holdeman et al., 1977).

$p H$ determinations on sterile filtrates of the faecal or caecal emulsions were done with a $p \mathrm{H}$ meter (Model 38B, Electronic Instruments Ltd, Richmond, Surrey).

\section{Growth of C. difficile in faecal emulsions derived from healthy donors}

Five sets of experiments were performed. (1) Dilutions of faeces $(5,10,20$ and 40 -fold $w / v)$ in sterile distilled water were prepared from a healthy 32-year-old adult donor and were seeded with $C$. difficile strain B-1. (2) Six cytotoxigenic strains (B-1, P-1, T-1, MA, BL and BT) and two non-cytotoxigenic strains (M-1 and $\mathrm{K}-1)$ of $C$. difficile were separately seeded into 20 -fold dilutions of faecal emulsion derived from a healthy 32-year-old adult. (3) $C$. difficile strain B-1 was inoculated into 20-fold dilutions of separate faecal emulsions prepared from the stools of 13 different healthy adult donors (mean age 30.8 years, range 21-57 years). (4) Strain B-1 was inoculated into multiple samples collected from a single healthy adult (age 32-35 years) over a 3-year period, (5) $C$. difficile strain B-1 was inoculated into separate faecal emulsions from nine healthy children (mean age 19.9 months, range 7 months to 3 years), 10 healthy geriatric patients (mean age 79.4 years, range 71-92 years) and a total of 23 healthy neonates (mean age $4 \cdot 6$ days, range 3-8 days). The neonatal stools comprised 12 from breast-fed infants (mean age 4.7 days, range 3-8 days) and 11 from bottlefed infants (mean age 4.4 days, range 3-7 days). These were treated as separate groups for the purpose of comparison between them and other age groups. In addition, multiple stool samples were collected over a 2year period from a healthy infant that had been breastfed. In all cases, total viable and spore counts of $C$. difficile, cytotoxin titres, and $p \mathrm{H}$ measurements were determined on the faecal emulsions at 24-h intervals.

\section{Growth of C. difficile in faecal emulsions derived from patients on antibiotics}

Three sets of experiments were performed. (1) Stools from 28 patients (mean age 48.3 years, range 3-84 years) with AAD, which gave negative results in tests for $C$. difficile and its toxin, were diluted in sterile distilled water to give a suspension equivalent to a 20 -fold dilution of normal stool and seeded with $C$. difficile strain B-1. (2) $C$. difficile strain B-1 was inoculated into 10 faecal emulsions derived from the stools of nine non-hospitalised adults (mean age 48.7 years, range $23-83$ years) who were receiving antibiotics but who did not have diarrhoea. (3) Fourteen specimens from patients with AAD which were culture-positive for $C$. difficile (mean age 58.9 years, range 27 to 83 years), were diluted in sterile distilled water to give a suspension equivalent to $1: 20 \mathrm{w} / \mathrm{v}$ and samples were taken for total viable and spore count of the patient's own (indigenous) strain of $C$. difficile, for cytotoxin titres and $p \mathrm{H}$ measurement. The emulsions were incubated for $72 \mathrm{~h}$, sampled at 24-h intervals, and analysed as above. In all cases, stool from a healthy adult was included as a control.

Those faecal emulsions found to be inhibitory to $C$. difficile (see below for criteria) were subjected to an additional test. A further portion of stool was diluted as above, centrifuged at $12000 \mathrm{rpm}$ at $4^{\circ} \mathrm{C}$ for $45 \mathrm{~min}$, and filtered through $0 \cdot 45-\mu \mathrm{m}$ and $0 \cdot 20-\mu \mathrm{m}$ membrane filters to sterilise. The filtrate was seeded with $C$. difficile strain B-1 and survival of the organism monitored as above. Stool filtrate from a healthy adult was included as a control.

Growth of C. difficile in faecal emulsions derived from patients with diarrhoea unassociated with antibiotic therapy

Stools from 16 patients with diarrhoea (mean age $31 \cdot 4$ years, range 2-64 years) were diluted to give suspensions equivalent to 20 -fold w/v dilutions of normal stool. Each faecal emulsion was divided in half and one portion used to prepare a sterile filtrate as described above. $C$. difficile was inoculated into the faecal emulsions and their corresponding filtrates, and survival of the organism, cytotoxin titre and $p \mathrm{H}$ values were monitored. Pathogens isolated from these stools before inclusion in the study were a salmonella from one and campylobacters from two. The other stools yielded no recognised pathogens.

\section{Determination of the role of viable bacteria in inhibition of growth of $C$. difficile}

(a) Stool from a healthy adult was diluted as described above and divided into three portions. One portion was sterilised by autoclaving for $20 \mathrm{~min}$ at $121^{\circ} \mathrm{C}$ and $15 \mathrm{lb} /$ in. ${ }^{2}$; one was centrifuged at $12000 \mathrm{rpm}$ for $45 \mathrm{~min}$ and the supernate filtered through $0.45-\mu \mathrm{m}$ and $0.2-\mu \mathrm{m}$ membrane filters to sterilise; and one was untreated. Samples $(10 \mathrm{ml})$ of these preparations were seeded with $C$. difficile, and growth of $C$. difficile, cytotoxin titres and $p \mathrm{H}$ measurements monitored at 24-h intervals. (b) Faecal emulsions were prepared from seven stool samples-two from healthy adults, three from healthy bottle-fed neonates and two from patients with AAD-as described 
above. All had previously been shown to be inhibitory to growth of $C$. difficile. The emulsions were sterilised by centrifugation and filtration. The resultant pellets of faecal material were re-suspended in sterile faecal filtrate derived from the stool of a healthy volunteer and incubated anaerobically for $24 \mathrm{~h}$. Samples $(10 \mathrm{ml})$ of the re-suspended stool pellet and analogous sterile filtrates were seeded with $C$. difficile strain B-1, and survival of the organism, cytotoxin titres and $p \mathrm{H}$ values were monitored.

\section{Growth of $C$. difficile in caecal contents from untreated and antibiotic-treated hamsters.}

One hamster was given clindamycin phosphate (Upjohn Ltd, Crawley, W. Sussex) $5 \mathrm{mg}$ by intraperitoneal injection and two were left untreated. All the animals were housed individually in sterile isolator cages with sterilised food and bedding (Larson et al., 1980); 5 days later they were killed and their caecal contents were harvested and diluted 20 -fold $\mathrm{w} / \mathrm{v}$ in sterile distilled water. The caecal material obtained from the untreated hamsters was pooled and a portion was centrifuged at $12000 \mathrm{rpm}$ for $30 \mathrm{~min}$ and filtered through a $0 \cdot 20-\mu \mathrm{m}$ membrane filter. C. difficile strain B-1 was inoculated into 10-ml portions of the caecal emulsions from the treated hamster and from the untreated group and into the filtrate from the untreated group; all were incubated for $72 \mathrm{~h}$. Total bacterial and spore counts of $C$. difficile, cytotoxin titres and $p \mathrm{H}$ measurements were determined before incubation and at 24-h intervals.

Criteria by which faecal emulsions were designated inhibitory or non-inhibitory to the growth of $C$. difficile

Faecal emulsions were considered to be inhibitory if, after incubation for $48 \mathrm{~h}$, the total numbers of viable $C$. difficile were less than those present at inoculation of the sample before incubation, and non-inhibitory if the total numbers of viable $C$. difficile were unchanged or greater than those present at inoculation of the sample before incubation. A 48-h period was selected as this was the period during which maximum change occurred with respect to survival of $C$. difficile.

\section{Statistical analysis}

Indices of the ability of the different groups of faecal emulsions to support the growth of $C$. difficile were: (1) a change in the $\log _{10}$ numbers representing the total count after $48 \mathrm{~h}$; (2) a change in the $\log _{2}$ number representing the toxin titre at $48 \mathrm{~h}$; and (3) the differences between $\log _{10}$ numbers representing the total and spore counts at $48 \mathrm{~h}$.

Groups were compared with each other for each of these three indices by use of an algorithm described by Hill and Peto (1971) to carry out a permutation test, which resembles Wilcoxon's test but deals with the observations themselves instead of their ranks and gives exact results even in the presence of large numbers of ties.

\section{Biochemical profiles of faecal emulsions}

API-ZYM strips (API Laboratory Products Ltd, Basingstoke, Hampshire) were charged with sterile faecal filtrate, 2 drops/enzyme test, and incubated, developed and reactions recorded as instructed by the manufacturers. The stool filtrates were also analysed for the presence of volatile fatty acids (VFA) by gas-liquid chromatography (Holdeman et al., 1977).

\section{Results}

Growth of C. difficile in faecal emulsions derived from healthy subjects

Eight different strains of $C$. difficile (6 toxigenic and 2 non-toxigenic) were inhibited to the same extent when separately added to faecal emulsion obtained from a single healthy adult donor. Therefore one strain (B-1) was used for all further experiments. A similar degree of inhibition of $C$. difficile occurred in 5, 10,20 and 40-fold dilutions of normal stool in sterile distilled water.

Growth of C. difficile was inhibited in all 13 faecal emulsions derived from the stools of healthy adults, with a mean $10^{3}$-fold decrease in total counts during the first 48-h period of incubation (table I). The total numbers of recoverable $C$. difficile differed little between tests incubated for 48 or $72 \mathrm{~h}$ (fig. 1) or for 6 days. As the total count fell the number of spores of $C$. difficile increased, until at $72 \mathrm{~h}$ mostly spores remained (fig. 1). There was no significant change in $p \mathrm{H}$ values, which remained at their original values (mean $8 \cdot 0 \pm 0 \cdot 6 \mathrm{SD}$; range $7 \cdot 3-8 \cdot 6$ ). Three of the faecal emulsions were weakly inhibitory, and with one of these there was an increase in cytotoxin titre. There was no increase in cytotoxin titre with any of the other samples.

Twenty-eight stool samples from one healthy adult taken over a 3-year period all produced good inhibition of growth of $C$. difficile, although the degree of inhibition varied, with a mean decrease in number of viable $C$. difficile $\left(\log _{10} / \mathrm{g}\right.$ of stool) of 3.4 (range 1.4-5.7). This variation did not appear to be seasonal or related to diet.

To confirm that the inhibition noted was due to the presence of viable bacteria, $C$. difficile was grown in heat-sterilised or filter-sterilised faecal emulsions derived from healthy adults. These sterile faecal emulsions supported the growth of $C$. difficile (fig. 2; table I) with concomitant production of cytotoxin (table I), the titre of which continued to rise throughout the test period (fig. 2). The differences in total counts of $C$. difficile achieved in the sterile faecal material compared with untreated faecal emulsions were highly significant $(p<0.001)$ 
Table I. Survival of $C$. difficile and changes in cytotoxin titre after growth for $48 \mathrm{~h}$ in faecal emulsions from different healthy subject groups

\begin{tabular}{|c|c|c|c|c|c|c|}
\hline \multirow[b]{2}{*}{ Subject group (number) } & \multicolumn{2}{|c|}{$\begin{array}{l}\text { Change in number } \\
\left(\log _{10}\right) \text { of } C \text {. difficile }\end{array}$} & \multicolumn{2}{|c|}{$\begin{array}{c}\text { Change in } \log _{2} \text { cytotoxin } \\
\text { titre }\end{array}$} & \multicolumn{2}{|c|}{$\begin{array}{c}\text { Difference in total } \\
\text { viable count } \\
\text { and spore count }\left(\log _{10}\right)\end{array}$} \\
\hline & Mean $\pm S D$ & (Range) & Mean \pm SD & (Range) & Mean \pm SD & (Range) \\
\hline $\begin{array}{l}\text { Adults (13) } \\
\text { sterilised emulsion (7) }\end{array}$ & $\begin{array}{c}-2 \cdot 8 \pm 1 \cdot 5 \\
1 \cdot 5 \pm 1^{*}\end{array}$ & $\begin{array}{c}(-4 \cdot 2--0 \cdot 1) \\
(0 \cdot 7-3 \cdot 8)\end{array}$ & $\begin{array}{l}1 \cdot 3 \pm 2 \cdot 6 \\
5 \cdot 3 \pm 2 \cdot 7^{*}\end{array}$ & $\begin{array}{c}(0-9 \cdot 0) \\
(3 \cdot 0-10 \cdot 0)\end{array}$ & $\begin{array}{l}1 \cdot 1 \pm 2 \cdot 6 \\
2 \cdot 2 \pm 0 \cdot 8 \dagger\end{array}$ & $\begin{array}{r}(0-2 \cdot 6) \\
(1 \cdot 3-3 \cdot 7)\end{array}$ \\
\hline Geriatric patients $(10)$ & $-1 \cdot 7 \pm 1$ & $(-3 \cdot 1--0 \cdot 1)$ & $1 \cdot 0 \pm 0 \cdot 7$ & $(0-2 \cdot 0)$ & $1.4 \pm 1.0$ & $(0-3 \cdot 7)$ \\
\hline Children (9) & $-2 \cdot 0 \pm 1$ & $(-3 \cdot 7--0 \cdot 8)$ & $1 \cdot 4 \pm 1 \cdot 6$ & $(0-4 \cdot 0)$ & $2 \cdot 0 \pm 0 \cdot 4 \dagger$ & $(1 \cdot 2-2 \cdot 7)$ \\
\hline $\begin{array}{l}\text { Neonates (23) } \\
\text { breast-fed (12) } \\
\text { bottle-fed (11) }\end{array}$ & $\begin{array}{l}-2 \cdot 0 \pm 1 \cdot 7 \\
-2 \cdot 8 \pm 1 \cdot 3 \S \\
-1 \cdot 2 \pm 1 \cdot 8 \dagger\end{array}$ & $\begin{array}{l}(-4 \cdot 9-0 \cdot 4) \\
(-4 \cdot 1--0 \cdot 6) \\
(-4 \cdot 9-0 \cdot 4)\end{array}$ & $\begin{array}{r}1 \cdot 3 \pm 2 \cdot 7 \\
-4 \cdot 4 \pm 2 \cdot 7 \dagger \\
2 \cdot 6 \pm 3 \cdot 4 \ddagger\end{array}$ & $\begin{array}{l}(0-10 \cdot 0) \\
(-9-0) \\
(0-10 \cdot 0)\end{array}$ & $\begin{array}{l}1 \cdot 7 \pm 1 \cdot 4 \\
1 \cdot 3 \pm 1 \cdot 5 \\
2 \cdot 2 \pm 1 \cdot 0^{*}\end{array}$ & $\begin{array}{l}(0 \cdot 1-4 \cdot 0) \\
(0 \cdot 1-4 \cdot 0) \\
(0 \cdot 5-3 \cdot 8)\end{array}$ \\
\hline
\end{tabular}

* Significantly different from healthy adult group ( $p \gtrless 0.01)$.

$\dagger$ Significantly different from healthy adult group $(\mathrm{p}<0.05)$.

$\ddagger$ Significantly different from breast-fed group ( $p \gtrless 0.01$ ).

$\S$ Significantly different from bottle-fed group $(\mathrm{p}<0 \cdot 05)$.

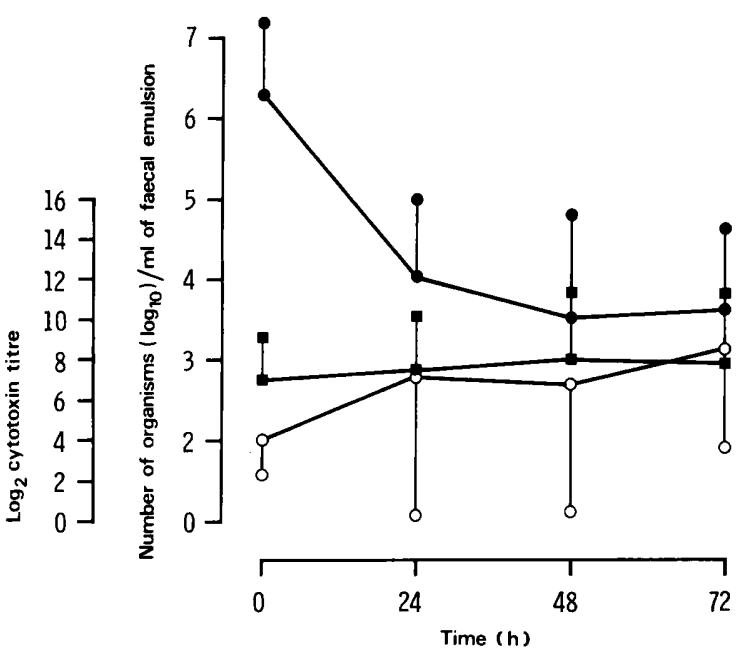

Fig. 1. Survival of $C$. difficile and cytotoxin production in faecal emulsions from healthy adults. Mean and SD for: $\square$ cytotoxin, total count, $O$ spore count.

(table I), as were the differences between cytotoxin titres for heat-sterilised faecal emulsions $(\mathrm{p}<0.01)$ and sterile faecal filtrates $(\mathrm{p}<0.05)$ compared with untreated faecal emulsions (table I) into which $C$. difficile was seeded. In the sterile faecal emulsions the numbers of spores gradually increased during incubation but were always $c .10^{2}$-fold lower than the total numbers of $C$. difficile during the first $48 \mathrm{~h}$ (fig. 2; table I). This difference between total and spore count at $48 \mathrm{~h}$ was significantly different from that for untreated faecal emulsions $(p<0.05)$.

Analysis of the growth of $C$. difficile in faecal emulsions derived from stools of healthy subjects in

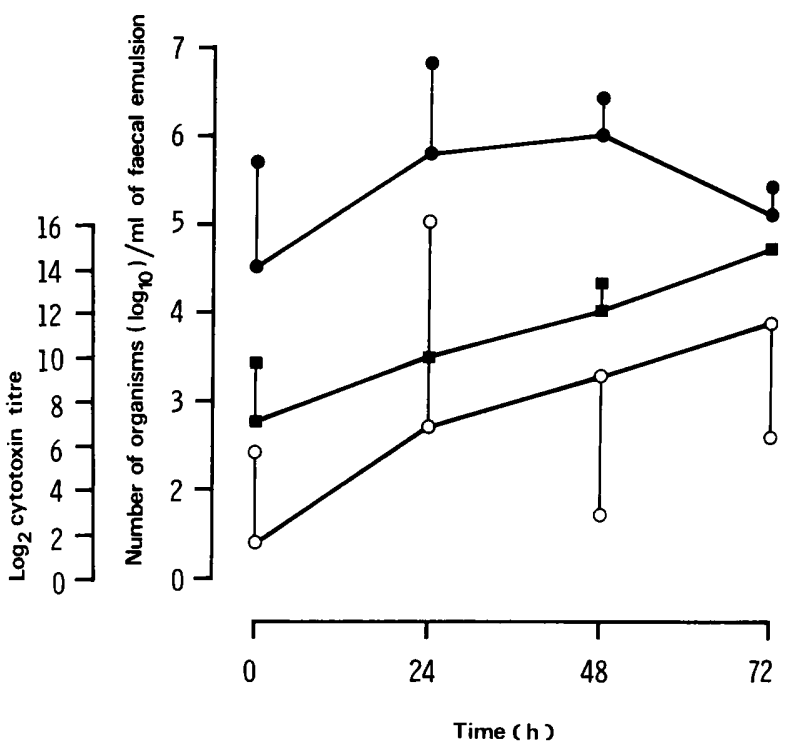

Fig. 2. Survival of $C$. difficile and cytotoxin production in sterilised faecal emulsions from healthy adults. For key, see fig. 1.

different age groups showed that those from geriatric patients, children and bottle-fed infants were generally less inhibitory than those from healthy adults (table I). These differences were only statistically significant when comparing healthy adults with bottle-fed infants for change in total numbers of $C$. difficile at $48 \mathrm{~h}(\mathrm{p}<0.05)$ and with bottle-fed infants and children for relative differences in viable count of $C$. difficile and spore count at $48 \mathrm{~h}(\mathrm{p}<0.01$ and $\mathrm{p}<0.05$ respectively). There was also a signifi- 
cant difference in total viable count of $C$. difficile in emulsions from breast and bottle-fed infants $(p<0.05$; table I). Although the faecal emulsions from breast-fed infants were more acidic (mean $p \mathrm{H}$ $5 \cdot 5 \pm 0.9 \mathrm{SD}$, range $4 \cdot 7-8 \cdot 2$ ) than those from bottlefed infants (mean $p \mathrm{H} 6.4 \pm 0.8 \mathrm{SD}$, range 5.8-7.8), inhibition of growth of $\bar{C}$. difficile could not be linked directly to $p \mathrm{H}$ as one of the inhibitory emulsions from a breast-fed infant had a $p \mathrm{H}$ value of $8 \cdot 2$.

There was a striking decrease in the amount of cytotoxin detected after growth in the faecal emulsions from the breast-fed infants when compared with bottle-fed infants $(\mathrm{p}<0.01)$ and healthy adults $(\mathrm{p}<0.05$; table I). The decrease of cytotoxin in the breast-fed group correlated with the acidic $p H$ values and is likely to have been due to denaturation as the toxin is unstable below $p H$ 5.5. This does not explain the apparent anomaly in the bottle-fed group in which one faecal emulsion with a $p \mathrm{H}$ value of 5.3 was extremely inhibitory to $C$. difficile and yet there was a $10^{3}$-fold increase in cytotoxin.

Repeated sampling from a breast-fed infant during the first 2 years of life showed a greater fluctuation in inhibitory capacity than with samples from an adult during the same period. This variability was particularly evident during the first week of life (fig. 3). Samples obtained at 2, 4 and 5 days of age exhibited good inhibition but those obtained on days 6,7 and 8 were non-inhibitory towards $C$. difficile. From 2 weeks to 2 years of age, the samples were always inhibitory, although very weak inhibition was noted at 3 weeks and 6 months (fig. 3 ). Throughout breast-feeding the $p \mathrm{H}$ remained low $(5 \cdot 4-5 \cdot 8)$ and the advent of mixed feeding did not markedly affect inhibition. Once the infant was fully weaned the faecal emulsions became alkaline (mean $p \mathrm{H} \mathrm{7.8)}$ and inhibitory to the same extent as those from healthy adults.

Growth of $C$. difficile in faecal emulsions from patients with $A A D$ not due to $C$. difficile

Six of the 28 samples $(21.4 \%)$ were non-inhibitory to the growth of $C$. difficile, 13 were extremely inhibitory, and nine were only weakly inhibitory (fig. 4). This inhibition was not related to $p \mathrm{H}$. Only one sample gave a value of below $p \mathrm{H} 6.0$ and the mean value of $p \mathrm{H} 8.0 \pm 0.8 \mathrm{SD}$ was the same as that for the healthy adult group. The inhibitory capacity of the AAD specimens was lower than for those from healthy adults (table II), but the difference was not statistically significant. However, the range of inhibition was much greater in the AAD group. An attempt was made to determine the extent to which inhibition was due to the resident microbial flora or residual antibiotic in the sample. This was achieved by monitoring the growth of $C$. difficile in sterile filtrates obtained from the inhibitory faecal emulsions. All of these sterile filtrates were inhibitory, with a mean decrease in total numbers of viable $C$. difficile of $\log _{10} 3 \cdot 3 \pm 2 \cdot 6$ SD (range $7 \cdot 8-0 \cdot 1$ ). Thir-

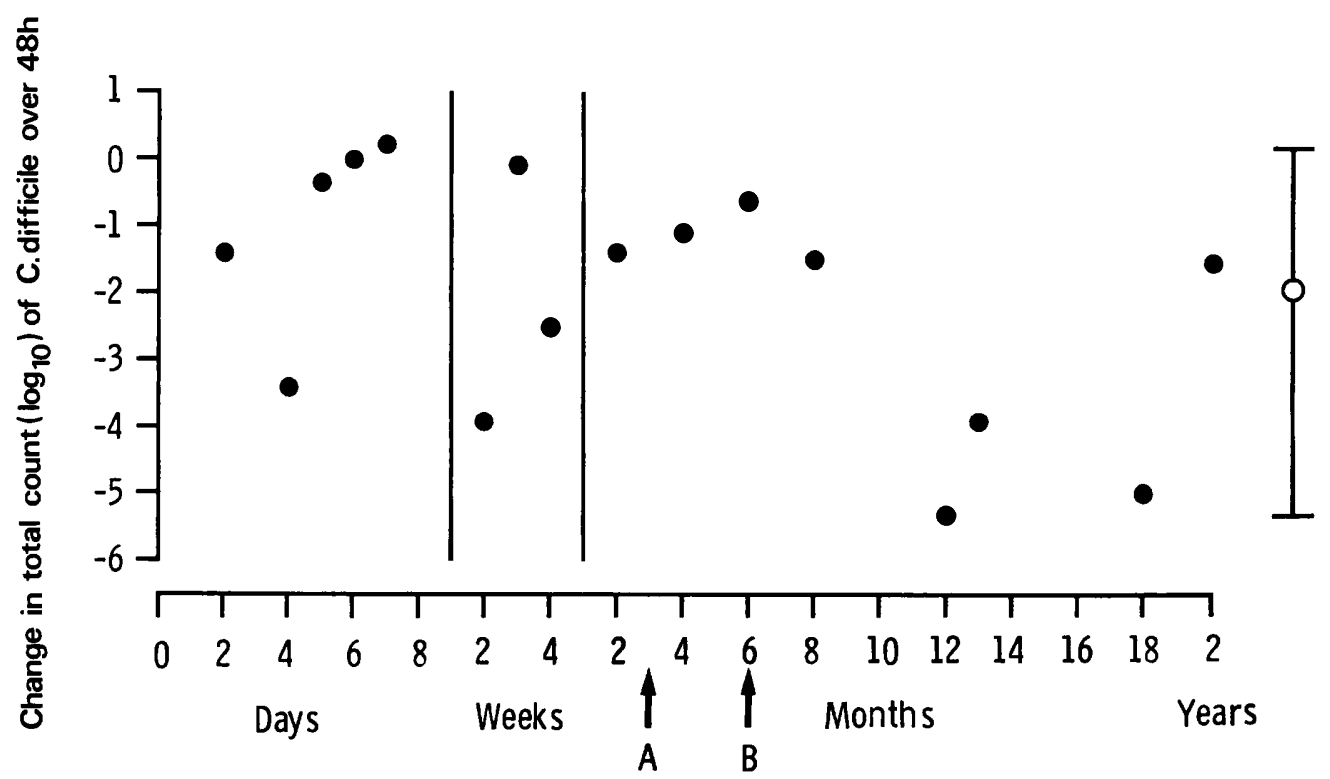

Fig. 3. Survival of $C$. difficile in multiple stool samples from a single healthy infant: $A=$ mixed feeding started; $B=$ fully weaned; $\mathrm{O}=$ mean; $\mathrm{I}=\mathrm{SD}$. 
teen of these filtrates were grossly inhibitory, and nine were weakly inhibitory. When inhibition by the faecal emulsion was greater than that shown by the filtrate, it was assumed that this enhanced inhibition was due in part to the faecal flora. It is likely that where both emulsion and filtrate gave good inhibition, residual antibiotic in the sample contributed to the inhibition noted.

Growth of $C$. difficile in faecal emulsions from patients on antibiotics but who did not have diarrhoea

Three faecal emulsions from nine patients $(33.3 \%)$ were non-inhibitory and there was a corresponding large increase in cytotoxin titre. However, seven faecal emulsions from six subjects were inhibitory to $C$. difficile, and in each case the sterile faecal filtrates (mean $p \mathrm{H} 7 \cdot 2 \pm 0 \cdot 7 \mathrm{SD}$, range 5.97.9) were also inhibitory, in contrast to the findings with sterile faecal filtrates from healthy adults. A further difference was that in two of these faecal filtrates, both of which caused only about a 10 -fold decrease in viable numbers of $C$. difficile, there was a large increase in the cytotoxin titre. Only the relative difference between total viable numbers of $C$. difficile and numbers of its spores were significantly different $(\mathrm{p}<0.05)$ when comparing this subject group with healthy adult controls (table II).

Growth of indigenous $C$. difficile in culture-positive stools

In these cases, the faecal emulsions were not seeded with $C$. difficile, but the fate of the indige- nous strain was monitored. The $p \mathrm{H}$ values of these emulsions were: mean $7 \cdot 3 \pm 1 \cdot 0 \mathrm{SD}$, range $5 \cdot 9-8 \cdot 5$. In two of the faecal emulsions the numbers of $C$. difficile decreased over $48 \mathrm{~h}$, but in both cases at $72 \mathrm{~h}$ there was an overall increase. In all 12 of the remaining emulsions there was active growth. The

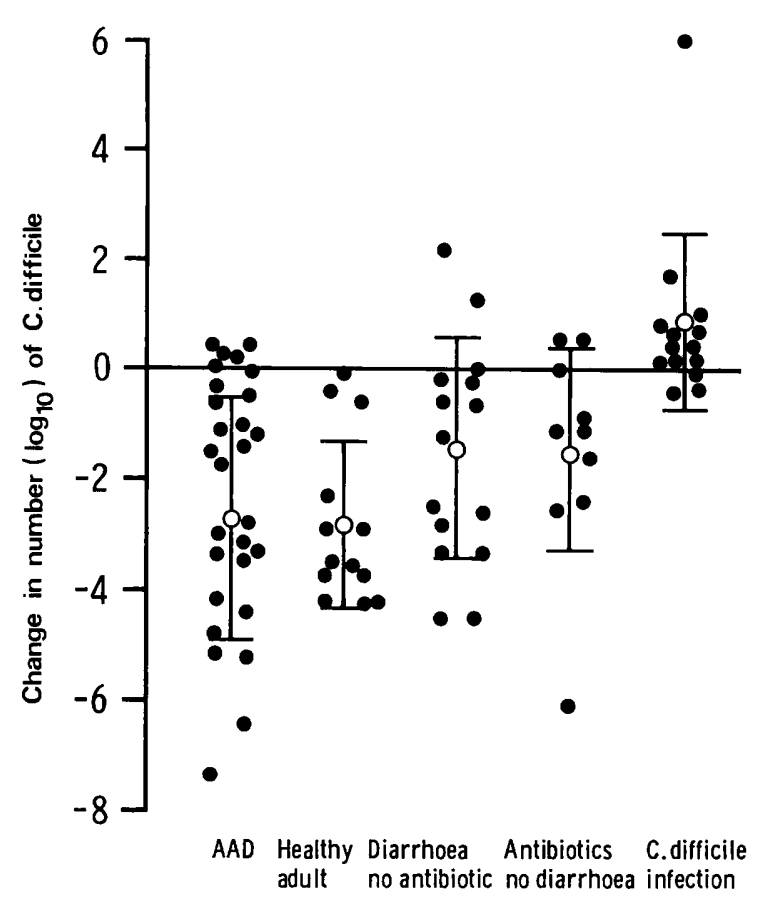

Fig. 4. Survival of $C$. difficile after incubation for $48 \mathrm{~h}$ in faecal emulsions from different patient groups and healthy subjects: $\mathrm{O}=$ mean, $\mathrm{I}=\mathrm{SD}$.

Table II. Comparison of survival of $C$. difficile and changes in cytotoxin titre after growth for $48 \mathrm{~h}$ in faecal emulsions from adult subjects in various groups

\begin{tabular}{|c|c|c|c|c|c|c|}
\hline \multirow[b]{2}{*}{ Subject group (number) } & \multicolumn{2}{|c|}{$\begin{array}{l}\text { Change in number } \\
\left(\log _{10}\right) \text { of } C \text {. difficile }\end{array}$} & \multicolumn{2}{|c|}{$\begin{array}{c}\text { Change in } \log _{2} \text { cytotoxin } \\
\text { titre }\end{array}$} & \multicolumn{2}{|c|}{$\begin{array}{c}\text { Difference in total } \\
\text { viable count } \\
\text { and spore count }\left(\log _{10}\right)\end{array}$} \\
\hline & Mean $\pm S D$ & (Range) & Mean \pm SD & (Range) & Mean \pm SD & (Range) \\
\hline Healthy adults (13) & $-2 \cdot 8 \pm 1.5$ & $(-4 \cdot 2--0 \cdot 1)$ & $1 \cdot 3 \pm 2 \cdot 6$ & $(0-9 \cdot 0)$ & $1 \cdot 1 \pm 2 \cdot 6$ & $(0-2 \cdot 6)$ \\
\hline $\begin{array}{l}\text { Antibiotic-associated } \\
\text { diarrhoea }(28)\end{array}$ & $-2 \cdot 8 \pm 2 \cdot 2$ & $(-7 \cdot 3-0 \cdot 4)$ & 20120 & $(0-7 \cdot 0)$ & $1.5 \pm 1.4$ & $(0-5 \cdot 7)$ \\
\hline $\begin{array}{l}\text { Diarrhoea unassociated } \\
\text { with antibiotics (16) }\end{array}$ & $-1 \cdot 4 \pm 2 \cdot 0$ & $(-4 \cdot 5-2 \cdot 2)$ & $3 \cdot 2 \pm 4 \cdot 2$ & $(0-11 \cdot 0)$ & $1 \cdot 2 \pm 1 \cdot 1$ & $(0-3 \cdot 3)$ \\
\hline $\begin{array}{l}\text { Antibiotic usage but } \\
\text { no diarrhoea (9) }\end{array}$ & $-1 \cdot 5 \pm 1 \cdot 9$ & $(-6 \cdot 0-0 \cdot 6)$ & $3 \cdot 7 \pm 4 \cdot 1$ & $(0-10 \cdot 0)$ & $2.8 \pm 1.9 \dagger$ & $(0-6 \cdot 3)$ \\
\hline $\begin{array}{l}\text { C. difficile-infected } \\
\text { patients (14) }\end{array}$ & $0.9 \pm 1.6 \S$ & $(-0.4-6.0)$ & $0.4 \pm 0.8$ & $(0-2 \cdot 0)$ & $1 \cdot 8 \pm 1 \cdot 7$ & $(0-4 \cdot 9)$ \\
\hline
\end{tabular}

$† \S$ See Table I for key. 
growth of $C$. difficile in this group was significantly greater when compared with its growth in faecal emulsions from healthy adults $(p<0.001)$.

Growth of C. difficile in faecal emulsions derived from patients with diarrhoea unassociated with antibiotics

Three of 16 specimens $(18.7 \%)$ were non-inhibitory to $C$. difficile and two of the three sustained good growth of $C$. difficile, matching that seen in sterilised faecal emulsions and exceeding that achieved in AAD faecal emulsions. The mean $p H$ value of these specimens was: $7 \cdot 3 \pm 0 \cdot 8 \mathrm{SD}$, range 6.3-7.9. The remaining 13 specimens (mean $p \mathrm{H}$ $6 \cdot 6 \pm 1 \cdot 1 \mathrm{SD}$, range $5 \cdot 3-8 \cdot 6$ ) were as inhibitory as those from healthy adults. With four of five specimens that were weakly inhibitory there was an increase in cytotoxin titre over $48 \mathrm{~h}$, a finding also noted with one of the weakly inhibitory faecal emulsions from a healthy adult. None of the differences for growth of $C$. difficile or cytotoxin production was statistically significant (table II).

An association between samples from patients with diarrhoea which allowed growth of $C$. difficile in vitro and the presence of other enteric pathogens was not apparent.

Nine of 15 sterile filtrates prepared from 12 inhibitory and three non-inhibitory faecal emulsions supported the growth of $C$. difficile with concomitant rises in cytotoxin titre. The six inhibitory filtrates were derived from inhibitory emulsions. Four of these filtrates had $p \mathrm{H}$ values of 5.5 and below, an acidity at which growth of $C$. difficile is inhibited (Borriello and Barclay, unpublished observations). The remaining two inhibitory filtrates were of neutral $p \mathrm{H}$; a small increase in cytotoxin was detected in both.

\section{The relative roles of viable bacteria and other antibacterial substances}

In tests with those AAD specimens from which both emulsion and filtrate were extremely inhibitory, an attempt was made to determine whether or not the microbial component alone would also inhibit $C$. difficile. Two such faecal emulsions were centrifuged and the bacterial pellet re-suspended in a heat-sterilised faecal emulsion derived from a healthy adult. In both cases the re-suspended material was inhibitory though to a much lesser extent than the original faecal emulsion or filtrate. This implies that a sufficient component of the faecal flora was present in each case to inhibit $C$. difficile, although it is also possible that an inhibi- tory amount of antibiotic was carried over in the microbial pellet.

The above test was also performed on two inhibitory faecal emulsions derived from healthy adults whose sterile filtrates were non-inhibitory, and three derived from healthy bottle-fed neonates whose filtrates were acidic and very inhibitory. When the microbial components of each of these emulsions were re-suspended in sterile faecal filtrate from an adult donor, the microbial components derived from healthy adults retained their inhibitory capacity, whereas the re-suspended material from two of the three neonatal emulsions were only weakly inhibitory and one was non-inhibitory.

\section{Growth of $C$. difficile in hamster caecal contents}

The in-vitro test for colonisation resistance to $C$. difficile infection based on growth of the organisms in faecal emulsions was performed on material from untreated hamsters and from clindamycin-treated hamsters which are extremely susceptible to $C$. difficile infection for at least 15 days after receiving clindamycin (Borriello and Barclay, 1985). Caecal emulsions from untreated hamsters inhibited $C$. difficile, with $10^{5}$-fold decreases in total viable counts and no increases in cytotoxin, whilst the corresponding filtrate allowed growth of the organism with a concomitant 32-fold increase in cytotoxin titre. This was the same pattern as that found in tests with faecal emulsions and filtrates from healthy people. The caecal emulsion prepared from the clindamycin-treated hamster was non-inhibitory and allowed a 10 -fold increase in total viable numbers of $C$. difficile and a 500 -fold increase in cytotoxin titre.

\section{Biochemical markers of colonisation resistance}

We found no enzyme marker that distinguished between faecal emulsions supporting the growth of $C$. difficile and those inhibiting growth. There was some variation in enzyme profiles, but this was evident even within the same subject group.

No correlation was found between the qualitative VFA profile of a faecal emulsion and the ability to inhibit the growth of $C$. difficile. Each of the stools from healthy adults and from patients with $C$. difficile-associated diarrhoea contained all the VFAs in the range $\mathrm{C} 1-\mathrm{C} 6$. In general, these were present in greater amounts than in the faecal emulsions from other groups. $C$. difficile itself produces each of these fatty acids, and this is likely to account for the findings in the faecal emulsions from patients with $C$. difficile infection. In general, 
the stool filtrates studied also contained most of the VFAs in the range $\mathrm{C}_{1}-\mathrm{C}_{6}$.

\section{Discussion}

Both growth and cytotoxin production by $C$. difficile are generally inhibited in faecal emulsions derived from the stools of healthy adults; if the faecal emulsions are sterilised either by filtration or autoclaving, the inhibition is lost and the growing organisms are metabolically active with cytotoxin produced and released. Thus, all of the requirements necessary for growth and cytotoxin production by $C$. difficile are present in the faecal emulsion, and inhibition seems to depend on the presence of viable bacteria. The parallel loss of inhibitory capacity when an active emulsion is filter-sterilised supports this conclusion. This inhibition was a consistent finding in analyses of multiple samples collected during a 3-year period from the one healthy adult studied in this way.

Although the vegetative cells of $C$. difficile are killed in the presence of the normal faecal flora, no sporicidal activity was seen. The number of spores increased slightly during the incubation period, indicating that a small portion of the vegetative cells in the initial inoculum had sporulated. In view of the demonstration by Ketley et al. (1984) of a rise in extracellular cytotoxin thought to be due to release by lysis, during the decline phase of $C$. difficile growth in vitro, we were surprised that with so much cell death there was no increase in extracellular cytotoxin in the majority of these faecal emulsions. It is unlikely that the constant level of cytotoxin detected was due to a continuous cycle of cytotoxin degradation and replenishment, because the cytotoxin is stable in faecal emulsions from healthy adults (Borriello and Barclay, unpublished observations).

When growth of $C$. difficile in faecal emulsions derived from stools from different age groups was compared, those from geriatric patients, children and bottle-fed neonates were less capable of inhibiting $C$. difficile than those from healthy adults. The emulsions derived from breast-fed neonates had inhibitory capacities comparable with those from healthy adults, but were significantly more inhibitory than those from bottle-fed infants. This difference between the two infant feeding groups could not be directly linked to faecal $p \mathrm{H}$, although the faecal emulsions from the breast-fed infants were more acidic. It is possible that breast-milk factors such as lactoferrin and secretory $\operatorname{IgA}$ were present in sufficient quantities in these stools to contribute to inhibition. The microbial flora obtained from acidic inhibitory faecal emulsions from bottle-fed neonates failed to inhibit, or poorly inhibited, $C$. difficile when re-suspended in sterilised faecal emulsions from a healthy adult donor; this implies that faecal $p \mathrm{H}$ was probably the most important inhibitory factor in these infants' stools.

Formula feeding has been shown to enhance intestinal colonisation by $C$. difficile in healthy infants (Cooperstock et al., 1982 and 1983). A higher carriage rate for bottle-fed infants has also been demonstrated for infants between the ages of 1 week and 4 months (Borriello and Barclay, 1984). Interestingly, we have shown that $13 \%$ of infant faecal emulsions, each from bottle-fed infants, supported the growth of $C$. difficile and cytotoxin production, indicating that these infants may have been susceptible to infection had they been exposed to the organism at the time of sampling.

Analysis of multiple samples from a breast-fed infant demonstrated that consistent inhibition of $C$. difficile only occurred after the infant had been fully weaned. This finding corresponds with the fact that infant carriage of $C$. difficile decreases with age, reaching about $7 \%$ when weaning has commenced (Borriello and Barclay, 1984) and the gut flora becomes much more complex. Faecal specimens obtained from healthy children were as inhibitory to $C$. difficile as those from healthy adults. However, the faecal emulsions derived from those children between the ages of 7 and 14 months were less inhibitory than those from children aged 21 months and older.

Generally the faecal emulsions from healthy geriatric patients were less inhibitory than those from other healthy adults, which correlates with the higher asymptomatic carriage rate of $C$. difficile $(11.8 \%)$ observed in geriatric subjects not taking antibiotics (Borriello and Barclay, unpublished observations). This could be a refiection of agerelated changes in the gut flora of the elderly (EllisPegler et al., 1975; Yamagishi et al., 1976).

The majority of faecal emulsions from patients with AAD were inhibitory to $C$. difficile and this contrasts with our findings for patients with $C$. difficile-mediated diarrhoea from whom, as would be expected, the faecal emulsions were not inhibitory. In half of these AAD cases, sterile filtrates of the emulsions were also very inhibitory. It was assumed that this inhibition was at least partly due to residual antibiotics in the stool. The residual microbial pellet from two of these faecal emulsions inhibited $C$. difficile when re-suspended in sterilised faecal emulsions from a healthy adult donor; this implies that sufficient components of the faecal flora were still present, but it is also possible that 
antimicrobial substances were carried over in the microbial pellet. It is important to note that in all of these patients the AAD was due to causes other than $C$. difficile and it is therefore not surprising that most of these faecal emulsions were inhibitory to this organism. A few faecal emulsions from AAD patients were not inhibitory, and it is possible that if these patients were exposed to the organism they would have become colonised. Although an antibiotic-mediated alteration of the gut flora is the most likely explanation for this lack of inhibition, it could also be a secondary effect of the induced diarrhoea which can cause changes in the intestinal flora (Levison and Kaye, 1969; Gorbach et al., 1970 ). When monitoring the growth of $C$. difficile in faecal emulsions derived from patients with diarrhoea unrelated to antibiotics, we found that some of them were non-inhibitory; thus these changes could predispose to $C$. difficile infection. There is some evidence that this may be so from the work of Falsen et al. (1980) who showed that $C$. difficile carriage was relatively common in patients with diarrhoea associated with other pathogens.

Of the nine adult subjects who did not have diarrhoea but were taking antibiotics, six were taking antibiotics commonly associated with $C$. difficile-mediated diarrhoea, i.e., penicillin, ampicillin, flucloxacillin and cefuroxime (Borriello and Larson, 1981). This would be the group most likely to be at risk of $C$. difficile infection, and interestingly the faecal emulsions from half of these were noninhibitory to the growth of $C$. difficile. All of the other faecal emulsions and their corresponding filtrates were inhibitory. It is possible that faecal samples from some of the other subjects could have lost their inhibitory capacity at a point when the levels of active antibiotic had decreased and before the normal faecal flora had re-established.

Because of the problems of confirming that patients with non-inhibitory faecal emulsions would have become colonised if exposed to $C$. difficile, we used the hamster model to study this aspect. Hamsters are extremely susceptible to $C$. difficile infection after treatment with clindamycin (Borriello and Barclay, 1985), whereas hamsters that do not receive antibiotics do not become infected (Larson et al., 1980). It was clearly demon-

\section{REFERENCES}

Barclay F E, Borriello S P 1982 In vitro inhibition of C. difficile. European Journal of Chemotherapy and Antibiotics 2:155156.

Barclay F E, Borriello S P 1984 Clostridium difficile and colonization resistance. In: Borriello S P (ed) Antibiotic- strated that growth of $C$. difficile in caecal emulsions in vitro reflected the in-vivo situation in that caecal emulsions prepared from untreated hamsters inhibited growth of $C$. difficile and toxin production, whereas those from a clindamycin-treated hamster did not.

Not all patients on antibiotic therapy are susceptible to infection with $C$. difficile, and the prompt recognition of those patients specifically at risk would be valuable. Although this could possibly be achieved with our bioassay based on growth of $C$. difficile in faecal emulsions, it would be preferable if an easily detectable faecal marker that correlated with the bioassay could be identified. Unfortunately we could find no such marker. There was no correlation between the qualitative VFA profile or any enzymic activity of a faecal emulsion and its ability to inhibit the growth of $C$. difficile. This lack of an association does not seem to be consistent with the association between the concentrations of various VFAs and colonisation resistance to $C$. difficile infection in hamsters reported by Rolfe (1984). Although, in general, higher quantities of all of the VFAs were present in the stools from healthy adults, in our study none of the sterile filtrates from these stools, which would have contained all of these fatty acids, was inhibitory.

Investigations into the role of colonisation resistance in preventing the establishment of $C$. difficile in vivo have been limited and much of the work has utilised animal models which do not necessarily mimic the human situation (Wilson et al., 1981). The development of an in-vitro test system based on the growth of $C$. difficile and cytotoxin production in faecal emulsions has permitted an examination of mechanisms that may contribute to colonisation resistance and has provided a method by which this resistance can be measured with respect to $C$. difficile infection. The use of this model may facilitate the identification of the minimum microbial components necessary for colonisation resistance to $C$. difficile infection in man and allow the development of simpler and safer preparations than those presently used for bacteriotherapy (Bowden et al., 1981; Schwan et al., 1984) in troublesome cases of $C$. difficile infection.

associated diarrhoea and colitis. Martinus Nijhoff Publishers. The Hague, p79.

Bartlett J G, Chang T-W, Gurwith M, Gorbach S L, Onderdonk A B 1978 Antibiotic-associated pseudomembranous colitis due to toxin-producing clostridia. New England Journal of Medicine 298:531-534.

Borriello S P, Barclay F E 1984 Colonization resistance to 
Clostridium difficile infection. Microecology and Therapy 14:75-87.

Borriello S P, Barclay F E 1985 Protection of hamsters against Clostridium difficile ileocaecitis by prior colonisation with non-pathogenic strains. Journal of Medical Microbiology 19:339-350.

Borriello S P, Honour P 1981 Simplified procedure for the routine isolation of Clostridium difficile from faeces. Journal of Clinical Pathology 34:1124-1127.

Borriello S P, Larson H E 1981 Antibiotics and pseudomembranous colitis. Journal of Antimicrobial Chemotherapy 7 (Suppl A): 53-62.

Bowden T A, Mansberger A R, Lykins L E 1981 Pseudomembranous enterocolitis: mechanism of restoring floral homeostasis. American Surgeon 47:178-183.

Brettle R P, Poxton I R, Murdoch J M, Brown R, Byrne M D, Collee J G 1982 Clostridium difficile in association with sporadic diarrhoea. British Medical Journal 284:230-233.

Cooperstock M S, Steffen E, Yolken R, Onderdonk A 1982 Clostridium difficile in normal infants and sudden infant death syndrome: An association with infant formula feeding. Pediatrics 70:91-95.

Cooperstock M, Riegle L, Woodruff V W Onderdonk A 1983 Asymptomatic colonisation of infants with Clostridium difficile: influence of age, sex and diet. Journal of Clinical Microbiology 17:830-833.

Ellis-Pegler R B, Crabtree C, Lambert H P 1975 The faecal flora of children in the United Kingdom. Journal of Hygiene 75:135-142.

Falsen E, Kaijser B, Nehls L, Nygren B, Svedhem A 1980 Clostridium difficile in relation to enteric bacterial pathogens. Journal of Clinical Microbiology 12:297-300.

George R H, Symonds J M, Dimock F, Brown J D, Arabi Y, Shinagawa N, Keighley M R B, Alexander-Williams J, Burdon D W 1978 Identification of Clostridium difficile as a cause of psuedomembranous colitis. British Medical Journal 1: 695 .

George W L, Sutter V L, Citron D, Finegold S M 1979 Selective and differential medium for isolation of Clostridium difficile. Journal of Clinical Microbiology 9: 214-219.

Gorbach S L, Banwell J G, Jacobs B, Chatterjee B D, Mitra R, Brigham K L, Neogy K N 1970 Intestinal microflora in Asiatic cholera. 1. "Rice-water" stool. Journal of Infectious Diseases 121: 32-37.
Hill I D, Peto R 1971 Probabilities derived from finite populations. Applied Statistics 20: 99-105.

Holdeman L V, Cato E P, Moore WEC (eds) 1977 Anaerobe laboratory manual, 4th edn. Virginia Polytechnic Institute and State University, Blacksburg, VA.

Ketley J M, Haslam S C, Mitchell T J, Stephen J, Candy D C A, Burdon D W 1984 Production and release of toxins A and B by Clostridium difficile. Journal of Medical Microbiology 18:385-391.

Koransky J R, Allen S D, Dowell V R 1978 Use of ethanol for selective isolation of spore forming micro-organisms. Applied and Environmental Microbiology 35:762-765.

Larson H E, Price A B, Borriello S P 1980 Epidemiology of experimental enterocecitis due to Clostridium difficile. Journal of Infectious Diseases 142:408-413.

Larson H E, Price A B, Honour P, Borriello S P 1978 Clostridium difficile and the aetiology of pseudomembranous colitis. Lancet 2:1312-1314.

Levison M E, Kaye D 1969 Faecal Flora in man: effect of cathartic. Journal of Infectious Diseases 119:591-596.

Malamou-Ladas H, Tabaqchali S 1982 Inhibition of Clostridium difficile by faecal streptococci. Journal of Medical Microbiology 15:569-574.

Rolfe R D 1984 Role of volatile fatty acids in colonization resistance to Clostridium difficile. Infection and Immunity 45:185-191.

Rolfe R D, Helebian S, Finegold S M 1981 Bacterial interference between Clostridium difficile and normal faecal flora. Journal of Infectious Diseases 143:470-475.

Schwan A, Sjolin S, Trottestam U, Aronsson B 1984 Relapsing Clostridium difficile enterocolitis cured by rectal infusion of normal faeces. Scandinavian Journal of Infectious Diseases 16:211-215.

Wilson K H, Silva J, Fekety F R 1981 Suppression of Clostridium difficile by normal hamster cecal flora and prevention of antibiotic-associated cecitis. Infection and Immunity 34:626628.

Yamagishi T, Serikawa T, Morita R, Nakamura S, Nishida S 1976 Persistent high numbers of Clostridium perfringens in the intestines of Japanese aged adults. Japanese Journal of Microbiology 20:397-403. 